\title{
A Twenty-Minute Nap Boosts the Planning Domain of Executive Function in Sleep Deprived Late Adolescents
}

\author{
Kyungmo Kang ${ }^{1}$, Juheon Choi ${ }^{2}$, Hee-jin Hwang ${ }^{3}$, Dae Lim Koo ${ }^{4}$, Jong Seung Kim ${ }^{5}$, Bumjo Oh ${ }^{5}$ \\ ${ }^{1}$ Division of Education \& Training, Korea Cancer Center Hospital, Seoul, \\ ${ }^{2}$ Cheongju Immigration Detention Center Clinic, Ministry of Justice, Cheongju, \\ ${ }^{3}$ Department of Family Medicine, Catholic Kwandong University College of Medicine, Incheon, \\ ${ }^{4}$ Department of Neurology, SMG-SNU Boramae Medical Center, Seoul, \\ ${ }^{5}$ Department of Family Medicine, SMG-SNU Boramae Medical Center, Seoul, Korea
}

Received April 6, 2017

Revised June 15, 2017

Accepted June 16, 2017

Address for correspondence Bumjo Oh, MD, MPH

Department of Family Medicine,

SMG-SNU Boramae Medical

Center, 20 Boramae-ro 5-gil,

Dongjak-gu, Seoul 07061, Korea

Tel: +82-2-870-2682

Fax: +82-2-831-0714

E-mail: bo39@snu.ac.kr
Objectives: The positive effects of nap on cognitive function and memory have been researched intensively among children and adults. However, the relationship between afternoon nap and executive function among sleep deprived late adolescents has not been studied as much. Methods: Ten sleep deprived, otherwise healthy Korean high school students were recruited, and asked to fill out Pittsburgh Sleep Quality Index questionnaire. They were put to nap for $5 \mathrm{~min}$ on three days during the first week, then for 10, 20, and $30 \mathrm{~min}$ in the same protocol during the consecutive weeks. After a 1-h class, both the students themselves and their corresponding teachers evaluated the participation level of the participants. They were then divided into two groups, and were instructed to play iPad game 'Free Flow. One group took a nap while the other group was prohibited from taking a nap. Both groups were instructed to play the game again. This was repeated once every afternoon for three days. Results: The experiment showed that 20-min nap was superior in subjective evaluations to the other nap duration, and the mean game score differences before and after were significantly higher in the nap taking group after the 20-min nap when analyzed using non-parametric statistical method ( $p$-value 0.04 ). Conclusions: Therefore, 20 -min nap is likely to boost planning domain of executive function on sleep deprived late adolescents.

J Sleep Med 2017;14(1):18-22

\section{Introduction}

Late adolescence is an important stage of human development when physical and psychological development occurs, and an individual prepares to become a healthy member of adult society. Although sleep is an important factor for adolescent development, Korean adolescents display more significant sleep deprivation and more irregular sleep and wake patterns than other adolescents in nations previously studied, and its detrimental effects are just beginning to be understood. ${ }^{1-6}$

Many previous studies have revealed that napping has many positive effects, especially on memory, mood, attention, performance and general health conditions. ${ }^{7-11}$ However, these studies mostly focused on the beneficial effect of napping on children and healthy adults, not on late adolescents to whom

This is an Open Access article distributed under the terms of the Creative Commons Attribution Non-Commercial License (http://creativecommons.org/licenses/by-nc/4.0) which permits unrestricted non-commercial use, distribution, and reproduction in any medium, provided the original work is properly cited. these useful effects of napping could be highly advantageous during learning.

Our first interest was the optimal nap time, which has been controversial in many different studies, ranging from less than $10 \mathrm{~min}$ to $2 \mathrm{~h}^{12-15}$ Secondly, we were intrigued by the fact that executive function could be assessed using various computer games. ${ }^{16}$ We decided to study the optimal nap duration among sleep deprived, otherwise healthy Korean late adolescents and the effect of a nap on their planning domain of executive function by comparing their performance before and after a nap using an easily accessible iPad game.

\section{Methods}

\section{Recruitment and study design}

A survey was conducted on 10 healthy high school students aged 17. The participants were asked to fill the Pittsburg Sleep Quality Index (PSQI). Sleep duration was investigated through 
the questionnaire: 'During the last 7 days, what is the time of the bed and what is the time of the sleep usually?' And subjective sleep deprivation was evaluated by response to the question: 'Do you think that sleeping time in the last 7 days is enough to recover from fatigue?'

The study was conducted from May 1, 2014 to May 30, 2014. Their participation was based on informed consent. The SMGSNU Boramae Medical Center Institutional Review Board approved this study.

\section{Experiment 1: optimal sleep duration}

Students were put to nap for 5-min after lunch for three times during the first week and then for 10, 20, and $30 \mathrm{~min}$ in the same protocol during the consecutive weeks. After a 1-h class after lunch recess, evaluation forms which included subjective concentration level, whether the student drowsed off during the class, subjective participation level relative to other students, and whether it is advisable to take a nap during lunch recess were issued both to the participants and their corresponding teachers.

\section{Experiment 2: executive function change after nap}

The commercial iPad puzzle game "Free Flow" is a simple game in which players connect several same colored dots with lines by touch. Players connect the dots of the identical color while not crossing other lines, at the same time completely filling all of the square background. Time attack mode was administered, and the number of puzzle cleared during 1-min period was measured. The square background size was set to be $5 \times 5$ (Fig. 1 ).

The students were divided randomly into two five-people groups, and each group was instructed to play the iPad puzzle game "Free Flow" for 1-min, and their scores were recorded. Group A students took a nap for 20 min after lunch while group B students were prohibited from taking a nap. The duration of the nap was to be determined by Experiment 1 . Immediately after the nap, group A students were instructed to play the game again. On the other hand, group B students waited for the time for group A students to take a nap and performed the same game. This procedure was repeated three times, once a day for three consecutive days. The before-nap and after-nap game scores were recorded.

\section{Statistical analysis}

All analyses were conducted using IBM SPSS ${ }^{\mathrm{TM}}$ (IBM SPSS 22.0 for windows, SPSS. Inc., Chicago, IL, USA). Mann Whitney $U$ test was utilized as the data set did not follow normal distribution. $p$ values less than 0.05 were considered statistically significant.

\section{Results}

\section{Demographic data and characteristics of subjects prior to intervention}

The questionnaire revealed basic information about the participants. The average sleep duration was 4.68 hours. Except
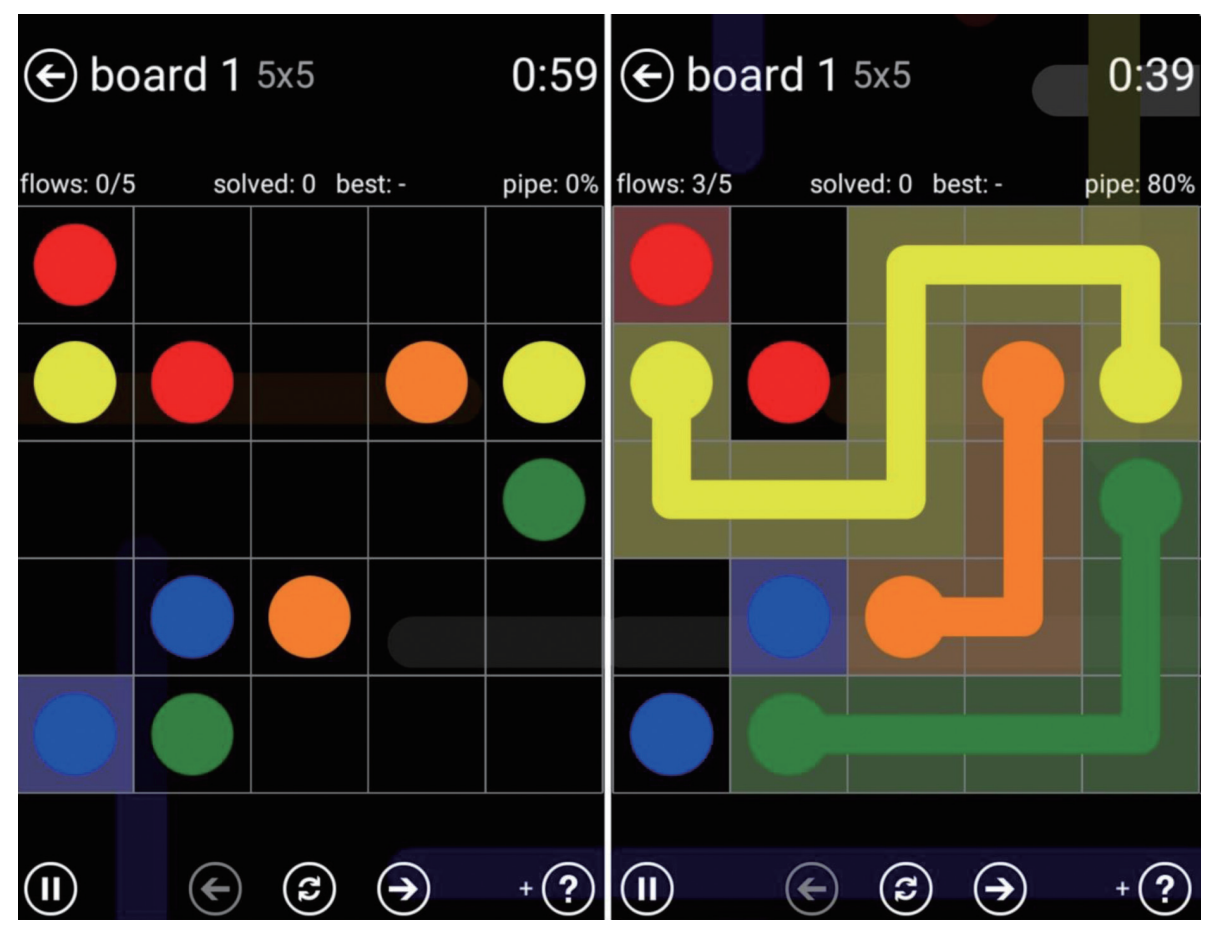

Figure 1. iPad game "Free Flow". 
for the absolute sleep deprivation which contributed each individual's PSQI total score of 2 or 3 (component 3: sleep duration), other PSQI component scores were all 0 . The participants' general information and sleep characteristics are summarized in Table 1.

\section{Optimal sleep duration}

The first experiment revealed that a 20-min nap was subjectively the most effective nap duration as judged by both the participants and their teachers. The specific evaluation result is summarized in Table 2, 3, Fig. 2, 3 is the graphical summary of the result.

Table 1. The participants' general information and sleep characteristics

\begin{tabular}{lc}
\hline \multicolumn{1}{c}{ Characteristics } & Test group $(\mathrm{n}=10)$ \\
\hline Age & All 17 years old \\
Sex & 8 \\
Female & 2 \\
Male & $4.68 \pm 0.57$ \\
Sleep duration (hours/day) & \\
Subjective sleep deprivations & 0 \\
Yes & 10 \\
No & \\
The quality of sleep & 10 \\
Very good & 0 \\
Good & 0 \\
Bad & 0 \\
Very bad & $1.5 \pm 1.12$ \\
Caffeine intake (times/week) & $2.56 \pm 0.52$ \\
PSQI score
\end{tabular}

Data are $\mathrm{n}(\%)$ or mean \pm standard deviation values

\section{Free Flow game score change after nap}

The average before-nap score and average after-nap score are displayed in Table 4. Five students in the A group who took $20 \mathrm{~min}$ of sleep had an average pre-sleep game score of 8.6 , and the average post-sleep time was 12.6 , which was improved by an average of 4 points. On the other hand, five students in the B group who did not take a nap had an average from 7.8 to 9.4 , which was improved by an average of 1.6 points. Mann-Whitney U test between group A and group B's before and after average game score difference showed statistically valid difference ( $p$-value 0.04 ). The score increase of nap-taking group was superior to the score increase of the group that did not take any nap during the experiment.

\section{Discussion}

According to the experiment conducted in this study, 20min nap was the most adequate duration according to the participants' and subjective evaluation. This finding was in accord with the subjective evaluation of the teachers who taught the participants during the classes after the nap. The 10-min nap was reported to be better than the 5-min nap, and a twentyminute- nap was reported to be superior to a 10 -min nap. However, a 30-min nap showed relatively poor evaluation results compared with 20-min nap. There was a statistically valid difference between group A and group B's game score difference between before-nap and after-nap situations. This was in accord with a previous study of recuperative effect of a nap of less than 30 min on subjective mood, visual detection performance, and symbol-digit substitution tasks. ${ }^{12}$ In addition, this study suggests that a 20-min nap after lunch could boost planning domain of executive function in late adolescents.

There have some studies assessing nap and brain functions,

Table 2. Participant's self evaluation result

\begin{tabular}{|c|c|c|c|c|c|}
\hline Question & Answer & $\begin{array}{l}\text { A (5 min }) \\
(n=10, \%)\end{array}$ & $\begin{array}{l}\mathrm{B}(10 \min ) \\
(\mathrm{n}=10, \%)\end{array}$ & $\begin{array}{l}\mathrm{C}(20 \min ) \\
(\mathrm{n}=10, \%)\end{array}$ & $\begin{array}{c}\mathrm{D}(30 \min ) \\
(\mathrm{n}=10, \%)\end{array}$ \\
\hline \multirow{3}{*}{$\begin{array}{l}\text { 1) The subjective participation and } \\
\text { concentration level during the class was }\end{array}$} & (1) Better than usual & 30 & 60 & 70 & 20 \\
\hline & (2) Same as usual & 40 & 40 & 30 & 30 \\
\hline & (3) Worse than usual & 30 & 0 & 0 & 50 \\
\hline \multirow[t]{2}{*}{ 2) Did you drowse off during the class? } & (1) Yes & 60 & 30 & 20 & 50 \\
\hline & (2) No & 40 & 70 & 80 & 50 \\
\hline \multirow{3}{*}{$\begin{array}{l}\text { 3) Compared with other students, how would } \\
\text { you evaluate your participation and } \\
\text { concentration level? }\end{array}$} & (1) Better than others & 20 & 80 & 90 & 30 \\
\hline & (2) Same as others & 40 & 20 & 10 & 10 \\
\hline & (3) Worse than others & 40 & 0 & 0 & 60 \\
\hline \multirow{4}{*}{$\begin{array}{l}\text { 4) Do you think it is advisable to take a nap } \\
\text { during lunch recess? }\end{array}$} & (1) Yes & 10 & 60 & 70 & 20 \\
\hline & (2) No & 60 & 20 & 10 & 40 \\
\hline & (3) I do not know & 30 & 20 & 20 & 40 \\
\hline & Total & 100 & 100 & 100 & 100 \\
\hline
\end{tabular}


Table 3. Teacher's evaluation of participants result

\begin{tabular}{|c|c|c|c|c|c|}
\hline Question & Answer & $\begin{array}{l}\text { A }(5 \min ) \\
(\mathrm{n}=3, \%)\end{array}$ & $\begin{array}{c}\mathrm{B}(10 \min ) \\
(\mathrm{n}=3, \%)\end{array}$ & $\begin{array}{l}\mathrm{C}(20 \mathrm{~min}) \\
(\mathrm{n}=3, \%)\end{array}$ & $\begin{array}{l}\mathrm{D}(30 \mathrm{~min}) \\
(\mathrm{n}=3, \%)\end{array}$ \\
\hline \multirow{3}{*}{$\begin{array}{l}\text { 1) The subjective participation and } \\
\text { concentration level of the students during } \\
\text { the class was }\end{array}$} & (1) Better than usual & 33.3 & 66.6 & 100 & 33.3 \\
\hline & (2) Same as usual & 33.3 & 33.3 & 0 & 66.6 \\
\hline & (3) Worse than usual & 33.3 & 0 & 0 & 0 \\
\hline \multirow{2}{*}{$\begin{array}{l}\text { 2) Did you see the participants drowse off } \\
\text { during the class? }\end{array}$} & (1) Yes & 66.6 & 33.3 & 0 & 66.6 \\
\hline & (2) $\mathrm{No}$ & 33.3 & 66.6 & 100 & 33.3 \\
\hline \multirow{3}{*}{$\begin{array}{l}\text { 3) Compared with other students, how would } \\
\text { you evaluate the students' participation and } \\
\text { concentration level? }\end{array}$} & (1) Better than others & 33.3 & 66.6 & 100 & 33.3 \\
\hline & (2) Same as others & 33.3 & 33.3 & 0 & 0 \\
\hline & (3) Worse than others & 33.3 & 0 & 0 & 66.6 \\
\hline \multirow{4}{*}{$\begin{array}{l}\text { 4) Do you think it is advisable to take a nap } \\
\text { during lunch recess? }\end{array}$} & (1) Yes & 33.3 & 66.6 & 66.6 & 33.3 \\
\hline & (2) $\mathrm{No}$ & 33.3 & 33.3 & 0 & 33.3 \\
\hline & (3) I do not know & 33.3 & 0 & 33.3 & 33.3 \\
\hline & Total & 100 & 100 & 100 & 100 \\
\hline
\end{tabular}

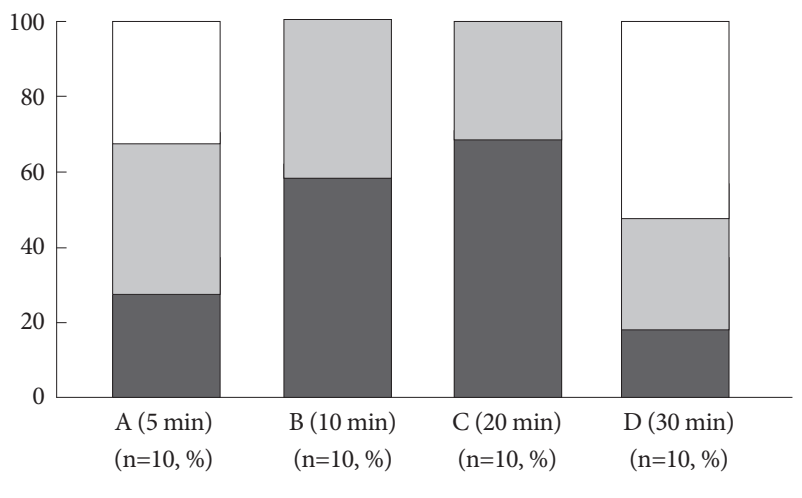

Better than usual $\quad \square$ Same as usual $\quad \square$ Worse than usual Figure 2. The subjective participation and concentration level during the class: students.

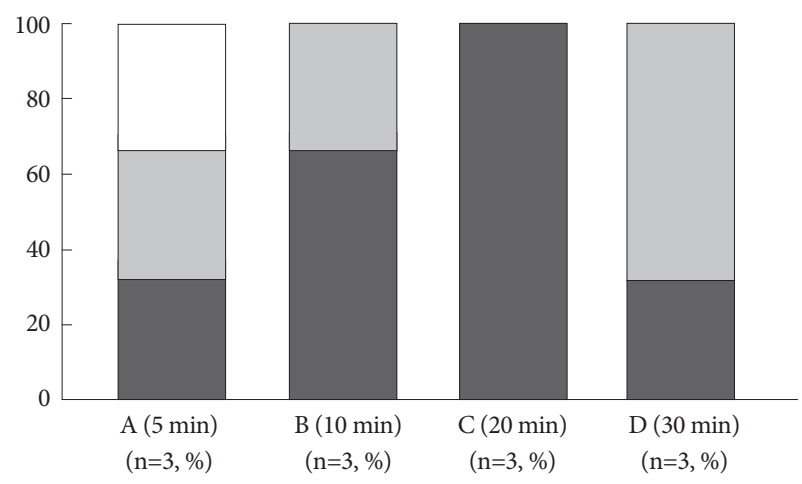

Better than usual $\quad \square$ Same as usual $\quad \square$ Worse than usual Figure 3. The subjective participation and concentration level during the class: teachers

including studies that opposed the called siesta culture's positive effect. ${ }^{17}$ However, our study results revealed that an after lunch nap among late adolescents has benefits on executive
Table 4. Comparison of game score result with group A and B

\begin{tabular}{ccccc}
\hline \multirow{2}{*}{ Group } & \multirow{2}{*}{ Student } & \multicolumn{3}{c}{ No. of solved puzzles (per 1 minute) } \\
\cline { 3 - 5 } & & Before & After & Difference \\
\hline \multirow{6}{*}{ A } & A-1 & 6 & 13 & 7 \\
& A-2 & 9 & 12 & 3 \\
& A-3 & 6 & 9 & 3 \\
& A-4 & 13 & 15 & 2 \\
& A-5 & 9 & 14 & 5 \\
& B-1 & 5 & 6 & 1 \\
& B-2 & 11 & 12 & 1 \\
& B-3 & 10 & 12 & 2 \\
& B-4 & 7 & 10 & 3 \\
& B-5 & 6 & 7 & 1 \\
\hline
\end{tabular}

function. To briefly summarize other studies on the benefits of naps, one study discovered the fact that $1 / 2 \mathrm{~h}$ or $2 \mathrm{~h}$ nap improves auditory reaction time and elevated activation compared with no nap, and the effect was similar between the two different durations of napping. ${ }^{13}$ Another study demonstrated that 30-min afternoon nap improves cognitive flexibility, a crucial component of executive function..$^{10}$ Finally, another study indicated that an ultra-short nap of less than $10 \mathrm{~min}$ is enough to promote declarative memory performance. ${ }^{15}$ The varied results on optimal nap duration may have been caused by age differences among the participant group (healthy adults versus late adolescents), and measured performance indices other than executive function. Compared with these studies, our study results provide supporting evidence on optimal nap time of less than 30-min in case of late adolescents.

There might be some limitations to our study. To list a few, students were not randomized during the first experiment 
on their duration of nap, and they were conditioned because they already knew how long they would take a nap before the experiment. In addition, although the participant groups show homogenous characteristics, they may not represent typical Korean late adolescents. Also, the number of test subject was only 10.

Additionally the iPad game "Free Flow" used in the experiment is not the gold standard method for evaluating planning domain of executive function. However, designing different tests sets in every test trial was very difficult to do, and using an identical test set over again was not advisable as the subjects may memorize the answer to the test. The reason we chose "Free Flow" as the measurement tool in this study was its great similarity to the "Unstructured Task" of "NIH EXAMINER battery", which was designed and verified to quantify subjects' planning domain of executive function. ${ }^{18}$

Despite these limitations, we conducted a simple pilot study on relatively homogenous late adolescents whose age was identical. This study is valuable as an original experimental attempt to demonstrate the effect of an afternoon nap on planning domain of executive function on sleep deprived late adolescents during a physical and psychological growth phase. We expect this study to be the basis of a larger scale study on the beneficial effect of napping on late adolescents, therefore establishing a positive role of napping in learning.

\section{Acknowledgments}

We would like to express our sincere gratitude to Minyoung Choi, Yerin Jee, Chaeyeon Kim and Cherin Lim, who inspired us to initiate this research work. They helped us collect the data that was crucial in deriving valuable insights and findings. This study would not have been made possible without their dedicated participation.

\section{REFERENCES}

1. Owens J; Adolescent Sleep Working Group; Committee on Adolescence. Insufficient sleep in adolescents and young adults: an update on causes and consequences. Pediatrics 2014;134:e921-e932.
2. Yang CK, Kim JK, Patel SR, Lee JH. Age-related changes in sleep/wake patterns among Korean teenagers. Pediatrics 2005;115(1 Suppl): 250256.

3. Louca M, Short MA. The effect of one night's sleep deprivation on adolescent neurobehavioral performance. Sleep 2014;37:1799-1807.

4. Asarnow LD, McGlinchey E, Harvey AG. The effects of bedtime and sleep duration on academic and emotional outcomes in a nationally representative sample of adolescents. J Adolesc Health 2014;54:350-356.

5. Carskadon MA. Sleep's effects on cognition and learning in adolescence. Prog Brain Res 2011;190:137-143.

6. Giedd JN. Linking adolescent sleep, brain maturation, and behavior. $J$ Adolesc Health 2009;45:319-320.

7. Debarnot U, Castellani E, Valenza G, Sebastiani L, Guillot A. Daytime naps improve motor imagery learning. Cogn Affect Behav Neurosci 2011; 11:541-550.

8. Horvàth K, Myers K, Foster R, Plunkett K. Napping facilitates word learning in early lexical development. J Sleep Res 2015;24:503-509.

9. Lemos N, Weissheimer J, Ribeiro S. Naps in school can enhance the duration of declarative memories learned by adolescents. Front Syst Neurosci 2014;8:103.

10. Slama H, Deliens G, Schmitz R, Peigneux P, Leproult R. Afternoon nap and bright light exposure improve cognitive flexibility post lunch. PLoS One 2015;10:e0125359.

11. Studte S, Bridger E, Mecklinger A. Nap sleep preserves associative but not item memory performance. Neurobiol Learn Mem 2015;120:84-93.

12. Hayashi M, Motoyoshi N, Hori T. Recuperative power of a short daytime nap with or without stage 2 sleep. Sleep 2005;28:829-836.

13. Taub JM, Tanguay PE, Rosa RR. Effects of afternoon naps on physiological variables performance and self-reported activation. Biol Psychol 1977; 5:191-210.

14. Lau EY, Wong ML, Lau KN, Hui FW, Tseng CH. Rapid-eye-movement-sleep (REM) associated enhancement of working memory performance after a daytime nap. PLoS One 2015;10:e125752.

15. Lahl O, Wispel C, Willigens B, Pietrowsky R. An ultra short episode of sleep is sufficient to promote declarative memory performance. J Sleep Res 2008;17:3-10.

16. Hagler S, Jimison HB, Pavel M. Assessing executive function using a computer game: computational modeling of cognitive processes. IEEE J Biomed Health Inform 2014;18:1442-1452.

17. Valencia-Flores M, Castaño VA, Campos RM, et al. The siesta culture concept is not supported by the sleep habits of urban Mexican students. J Sleep Res 1998;7:21-29.

18. Kramer JH, Mungas D, Possin KL, et al. NIH EXAMINER: conceptualization and development of an executive function battery. J Int Neuropsychol Soc 2014;20:11-19. 